\title{
Effet du niveau et de la nature des nutriments énergétiques sur la composition du lait et ses aptitudes technologiques : effet du monopropylène glycol
}

\author{
C Hurtaud, R Vérité, H Rulquin \\ avec la collaboration technique de B Marquis, $H$ Hétault, $S$ Marion, $L$ Toullec \\ INRA, station de recherches sur la vache laitière, 35590 Saint-Gilles, France
}

(Reçu le 19 août 1991; accepté le 22 octobre 1991)

\begin{abstract}
Résumé - L'effet positif de la nutrition énergétique sur le taux protéique peut être dû à une action intrinsèque du niveau d'apport et/ou à un changement dans la nature des nutriments énergétiques disponibles. Pour dissocier ces 2 mécanismes, on a testé par rapport à une ration atémoin négatif", l'effet sur la sécrétion des constituants du lait ( $3 \times 8$ vaches), d'un supplément d'énergie ne modifiant pas le profil fermentaire (témoin positif : supplément de ration complète, $+1,3 \mathrm{UFL}$ ) ou conduisant à un faciès propionique important par apport de 1,2 kg de monopropylène glycol (MPG, + 0,5 UFL). II n'y pas eu d'effet du niveau énergétique sur les paramètres mesurés. Le MPG a provoqué une diminution du taux butyreux $(-4 \mathrm{~g} / \mathrm{kg})$ et une augmentation du taux protéique $(+1 \mathrm{~g} / \mathrm{kg})$ sans modification de la production laitière. Ceci s'est traduit par une amélioration des qualités fromagères. Les mécanismes d'action du MPG sont discutés.
\end{abstract}

vache laitière / énergie / monopropylène glycol / lait / aptitude fromagère

Summary - Effect of level and nature of energetlc nutrients on milk composition and cheese-making: effect of propylene glycol. Energetic nutrition has a positive effect on milk protein contents; this may be due to an intrinsic effect of energy level or/and a change in the nature of the energetic nutrients. In this study, we attempted to dissociate these 2 mechanisms. The effect on milk production $(3 \times 8$ cows) of an energy supplement (+ 1.3 UFL) with no change in rumen profile ("positive control", an increase in the complete diet) or with more propionic acid due to ingestion of $1.2 \mathrm{~kg}$ of propylene glycol ("MPG") was compared with the effect of a "negative control" diet. The energy level was found to have no effect on milk composition. Milk fat (\%) was lower $(-4 \mathrm{~g} / \mathrm{kg})$ and milk protein (\%) higher $(+1 \mathrm{~g} / \mathrm{kg})$ with the propylene glycol supplement; there was no change in milk yield. Cheese-making was improved (coagulation time, cheese yield). The possible mechanisms of action of propylene glycol have been discussed, ie a change in rumen fermentation or a hormonal alteration.

dairy cow / energy / monopropylene glycol / milk / cheese-making efficiency 


\section{INTRODUCTION}

Pour augmenter le taux protéique du lait et améliorer les processus de la transformation fromagère, l'action sur la nutrition énergétique de la vache (régimes riches en concentré et/ou apports énergétiques accrus) est une voie connue depuis longtemps (Auriol et Jarrige, 1962). L'accroissement d'apport d'une UFL améliorerait en moyenne le taux et la quantité de protéines secrétées de $0,5 \mathrm{~g} / \mathrm{kg}$ et de $39 \mathrm{~g} / \mathrm{j}$ selon la revue de nombreux essais zootechniques de Remond (1985). Mais ces effets sont très variables et difficiles à contrôler, de même que les effets associés sur le taux butyreux et la physiologie ruminale.

L'amélioration de la sécrétion de protéines pourrait être due à un effet intrinsèque du niveau d'apport énergétique, mais aussi au changement de nature des fermentations et des acides gras volatils (AGV) dans le rumen, en particulier l'acide propionique. Des essais d'infusions d'acides gras volatils (Rook et Balch, 1961; Rook et al, 1965; Rulquin, 1982) montrent l'effet positif de l'acide propionique sur le taux protéique et l'effet négatif de l'acide acétique. Mais, dans la majorité des essais, l'augmentation des apports énergétiques entraîne une augmentation des apports azotés réels (apports microbiens, PDIM). De plus, les mécanismes sous-jacents, effet direct de la disponibilité mammaire en glucose, modifications de l'équilibre hormonal (insuline) et des sites d'utilisation, épargne métabolique de certains acides aminés, sont incertains.

L'importance respective des 2 hypothèses (effet du niveau ou de la nature des nutriments énergétiques) est à éclaircir et la recherche des voies pratiques permettant d'améliorer la fourniture d'acide propionique et de glucose est à poursuivre. Ceci peut être réalisé, entre autres, par l'apport de Monopropylène Glycol (MPG), utilisé jusqu'à présent pour prévenir ou traiter les cétoses en début de lactation chez la vache laitière (Remond et al, 1984). Ce produit est intéressant, car c'est un liquide qui peut être facilement mélangé à la ration des animaux et donc être ingéré sans problème. De plus, son effet sur les fermentations ruminales permet d'éviter les infusions d'acides gras volatils qui sont des méthodes délicates.

Nous l'avons utilisé dans cet essai de production sur vaches laitières pour dissocier l'effet intrinsèque du niveau énergétique de celui provoqué par le changement de nature du profil des nutriments. Pour cela, une ration de composition constante, distribuée en quantité limitée (témoin négatif) a été supplémentée (+ 2,2 UFL) sur une base isoénergétique et iso-azotée soit par un apport plus libéral du même régime ne changeant pas le faciès fermentaire (témoin positif), soit par un apport équivalent de $1,2 \mathrm{~kg}$ de MPG (régime MPG). Les effets sur les fractions protéiques et minérales du lait et ses propriétés technologiques ont été analysés.

\section{MATÉRIEL ET MÉTHODES}

\section{Traitement - Schéma expérimental - Alimentation}

Vingt-quatre vaches primipares Holstein d'un poids moyen de $565 \mathrm{~kg}$, environ à 3,5 mois de lactation (écart type de $20 \mathrm{j}$ ), alimentées individuellement avec une ration complète constante en matière sèche $(2 / 3$ d'ensilage de maïs, $1 / 3$ de concentré sur la base de la matière sèche) ont été réparties en 3 groupes aussi semblables que possible en fonction de leur production, leurs taux protéique et butyreux.

Trois rations ont été comparées selon un schéma en carré latin $3 \times 3$ avec des périodes de 3 semaines ( 1 semaine d'adaptation, 2 semaines de traitement) : 


\section{Une ration «témoin positif»}

Les quantités offertes représentaient $95 \%$ du niveau maximal d'ingestion de chaque vache (précédemment mesuré) afin de réduire au minimum les refus et de mieux contrôler les quantités ingérées.

\section{Une ration «MPG» (monopropylène glycol)}

Cette ration est obtenue en remplaçant sur une base isoénergétique, 2,2 UFL de la ration précédente par 1,2 kg de MPG.

\section{Une ration «témoin négatif»}

Les animaux reçoivent le même régime que le témoin positif, mais en quantité plus faible $(-2,2$ UFL soit environ $-15 \%$ ).

Les quantités distribuées à chaque vache ont été déterminées au début de l'essai. Afin de couvrir individuellement les besoins en azote et d'avoir des rations «iso-PDI", l'aliment "concentré" a été constitué d'une proportion variable selon les animaux et selon les traitements, de concentré énergétique et de tourteau tanné ( $80 \%$ de soja et $20 \%$ de colza). Chaque vache recevait en outre, $170 \mathrm{~g}$ d'urée et $250 \mathrm{~g}$ de complément minéral ( $12 \%$ de mélasse, $22 \%$ de calcium, $15 \%$ de phosphore, $3 \%$ de sodium, $3 \%$ de magnésium, $0,4 \%$ de soufre, 200000 $\mathrm{Ul} / \mathrm{kg}$ de vitamine $\mathrm{A}, 60000 \mathrm{Ul} / \mathrm{kg}$ de vitamine D3, $500 \mathrm{mg} / \mathrm{kg}$ de vitamine $\mathrm{E}, 550 \mathrm{ppm}$ de $\mathrm{Cu}$, $20 \mathrm{ppm}$ de $\mathrm{Co}, 80 \mathrm{ppm}$ de I, $2100 \mathrm{ppm}$ de $\mathrm{Mn}$, $2600 \mathrm{ppm}$ de $\mathrm{Zn}, 6 \mathrm{ppm}$ de Se). Les différents aliments, y compris le MPG, étaient mélangés et distribués, vache par vache, en bacs individuels à $9 \mathrm{~h}$ et à $17 \mathrm{~h}$.

Lors des transitions, l'apport de monopropylène glycol était accru ou diminué progressivement par tranche de $250 \mathrm{~g} / \mathrm{j}$.

\section{Mesures}

Les quantités d'aliment offertes et les refus éventuels étaient pesés individuellement chaque jour. La teneur en matière sèche des aliments a été mesurée chaque jour (fourrages) ou chaque semaine (concentré).
La quantité de lait produite était mesurée à chaque traite. Les taux de matières grasses, de protéines et de lactose étaient déterminés avec un analyseur infrarouge chaque semaine sur 6 échantillons par vache ( 6 traites). Les animaux étaient pesés chaque semaine.

Un prélèvement de sang a été effectué $1 \mathrm{~h}$ avant le repas du matin à chaque vache, le dernier mardi de chaque période. Les teneurs en urée (Talke et Schubert, 1965), en glucose (méthode a la glucooxydase; Trinder, 1969) et en bétahydroxybutyrate (Williamson et Mellanby, 1974) ont été déterminées sur le plasma déprotéinisé à l'acide perchlorique avec un analyseur automatique Isamat (ISA Biologie, $94230 \mathrm{Ca}$ chan).

Le $\mathrm{pH}$ et la teneur en acides gras volatils (AGV) du jus de rumen ont été mesurés entre 0 et $9 \mathrm{~h}$ après le repas du matin sur 3 vaches fistulées du rumen qui ont reçu successivement te régime "témoin positif" puis le régime MPG (mesures après 8 et 14 j d'adaptation).

\section{Caractéristiques chimiques et technologiques des laits}

Le dernier lundi de chaque période, $200-300 \mathrm{ml}$ de lait prélevés à la traite du matin sur chaque vache ont été écrémés immédiatement pour constituer des échantillons pondérés moyens par lot.

Les analyses ont été effectuées après écrémage et standardisation du $\mathrm{pH}$ à 6,61 (par adjonction d'acide chlorhydrique ou de soude) :

- matière sèche (passage à l'étuve à $100^{\circ} \mathrm{C}$ pendant $7 \mathrm{~h}$ );

- fractions azotées : azote total (méthode Kjeldahl), azote non protéique (précipitation par de l'acide trichloracétique $12 \%$ puis dosage de l'azote du filtrat), azote non caséinique (précipitation à pH 4,6 par de l'acide acétique et de l'acétate de sodium, puis dosage de l'azote du

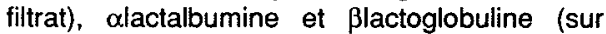
HPLC - High Performance Liquid Chromatograph par passage sur une colonne Tris, à partir du filtrat obtenu après la précipitation des caséines);

- minéraux ( $\mathrm{Ca}, \mathrm{Mg}, \mathrm{K}, \mathrm{Na}$ ) dosés sur le lait directement par spectrographie d'absorption. 
Les paramètres technologiques ont été mesurés au cours de la coagulation du lait à $35^{\circ} \mathrm{C}$ par action de la présure $(5,2 \mathrm{mg} /$ de chymosine) utilisée à la dose de $0,13 \mathrm{mg}$ de chymosine par litre de lait :

- évolution de la viscosité apparente lors de la phase initiale de la coagulation mesurée à l'aide du viscosimètre Rheomat 30 . Le liquide est cisaillé entre un cylindre fixe et un cylindre intérieur mobile tournant à un gradient de vitesse de $193 \mathrm{trs} / \mathrm{min}$. La viscosité est mesurée par la résistance opposée par le lait au cours de la coagulation sur la rotation du cylindre mobile (Korolczuck et Maubois, 1987);

- fermeté du caillé ( $2 \mathrm{~h}$ après emprésurage) : elle a été mesurée avec un analyseur de texture Stevens LFRA, par la force à appliquer à un cylindre de $12 \mathrm{~mm}$ de diamètre s'enfonçant à une vitesse de $0,5 \mathrm{~mm} / \mathrm{s}$ (Thomasow et Voss, 1977):

- «rendement fromager " en frais : $100 \mathrm{ml}$ de lait sont emprésurés et mis dans une étuve à $35^{\circ} \mathrm{C}$. Le décaillage est réalisé $90 \mathrm{~min}$ après l'emprésurage et est suivi immédiatement par une centrifugation à $2700 \mathrm{~g}$ pendant $10 \mathrm{~min}$. Le culot constitué par le caillé est pesé. le “rendement fromager» en frais est le rapport entre le poids du caillé et le poids du lait.

\section{Analyses statistiques}

L'analyse de variance pour le schéma en carré latin a été effectuée sur les données individuelles $(n=24)$ pour les paramètres zootechniques (lait, taux protéique, taux butyreux, lactose et paramètres sanguins) (seuil $5 \%$ ) et sur les données moyennes des traitements $(n=9)$ pour les paramètres biochimiques et technologiques (seuil $10 \%$ ). Le modèle était de la forme suivante : var $=A_{j}+B_{j}+C k+e\left(A_{i}=\right.$ vache, $i=1 \ldots 24$, ou $A_{i}=$ lot, $i=1 \ldots 3 ; B_{j}=$ période, $j=1 . . .3 ; C k=$ traitement, $k=1 . . .3 ; e=$ résidu).

\section{RÉSULTATS}

\section{Effet du niveau énergétique}

Avec le témoin positif, l'augmentation de l'apport énergétique, plus faible que pré- vue (1,3 UFL au lieu de 2,2 UFL) par suite de refus alimentaires, n'a modifié ni la production de lait, ni les teneurs en matières grasses, protéines et lactose (tableaux I et II).

De la même façon, elle n'a pas modifié la part des différentes fractions azotées du lait (tableau II) ni les caractéristiques de la phase active de la coagulation (vitesse de coagulation et évolution de la viscosité pendant la gélification) et de la fin de la coagulation (fermeté du caillé et "rendement fromager" en frais) (tableau III).

La teneur plasmatique en glucose a significativement augmenté et celle en urée a fortement diminué (-18\%) (tableau IV).

\section{Effet de l'apport de monopropylène glycol}

Cet effet sera apprécié par rapport à la moyenne des 2 témoins puisque ceux-ci n'ont pas présenté de différences.

L'apport de MPG n'a pas affecté la valeur moyenne (ni l'évolution postprandiale) du $\mathrm{pH}$ et de la teneur totale en AGV dans le rumen. Par contre, il a très fortement réduit la proportion moyenne d'acide acétique $(-12 \%)$ et augmenté celle d'acide propionique $(+5,3 \%)$ de sorte que le rapport C2/C3 a été seulement de 2,25 (vs 3,4). En outre, la proportion de chacun des AGV mineurs $\left(\mathrm{IC}_{4}, \mathrm{C}_{5}, \mathrm{IC}_{5}\right.$ et $\left.\mathrm{C}_{6}\right)$ a doublé pour représenter ensemble $7,8 \%$. Ces différences ont existé en permanence au cours de la journée mais atteignaient leur maximum seulement $6-9 \mathrm{~h}$ après le repas pour tous les AGV à l'exception de l'acide isobutyrique dont le pic était plus fugace et plus précoce (entre 1 et $3 \mathrm{~h}$ ) (tableau $\mathrm{V}$ et fig 1).

L'apport de monopropylène glycol n'a eu aucun effet sur la quantité de lait sécrétée mais a entraîné une modification im- 
Tableau I. Apports alimentaires et bilans nutritifs.

\begin{tabular}{|c|c|c|c|c|}
\hline \multirow{2}{*}{$\begin{array}{l}\text { Traitement } \\
\text { Niveau énergétique } \\
\text { Nature du supplément }\end{array}$} & \multirow{2}{*}{$\frac{\text { Témoin négatif }}{\text { Limité aux besoins }}$} & \multirow{2}{*}{$\frac{\text { Témoin positif }}{+2 \text { UFL }}$} & \multirow{2}{*}{$\begin{array}{r}M P G \\
+2 U F L \\
M P G\end{array}$} & \multirow[t]{2}{*}{ ETR } \\
\hline & & & & \\
\hline \multicolumn{5}{|l|}{ Quantités ingérées ( $\mathrm{kg}$ de MS) } \\
\hline Ensilage de maïs (a) & 10,5 & 11,3 & 10,2 & 0,1 \\
\hline Concentré énergétique (b) & 3,8 & 5,1 & 3,8 & 0,06 \\
\hline Tourteau tanné (c) & 1,1 & 0,5 & 1,1 & 0,02 \\
\hline Urée & 0,170 & 0,170 & 0,170 & - \\
\hline MPG & 0,0 & 0 & 1,2 & - \\
\hline \multicolumn{5}{|l|}{ Apports nutritifs } \\
\hline MAT $(g)$ & 149 & 133 & 151 & 10 \\
\hline UFL & 14,0 & 15,3 & 16,0 & 0,1 \\
\hline PDIN (g) & 1517 & 1457 & 1508 & 11 \\
\hline PDIE (g) & 1505 & 1460 & 1492 & 14 \\
\hline \multicolumn{5}{|l|}{ Bilans nutritifs } \\
\hline Bilan UFL & 0,1 & 1,4 & 0,5 & 0,2 \\
\hline Bilan PDI & 107 & 81 & 166 & 13 \\
\hline
\end{tabular}

Les caractéristiques nutritives des aliments sont les suivantes (par $\mathrm{kg}$ de MS) : (a) : 0,87 UFL, $50 \mathrm{~g}$ de PDIN, $71 \mathrm{~g}$ de PDIE, $76 \mathrm{~g}$ de MAT; (b) : 0,97 UFL, $84 \mathrm{~g}$ de PDIN, $95 \mathrm{~g}$ de PDIE, $127 \mathrm{~g}$ de MAT; (c) : 1,13 UFL, $389 \mathrm{~g}$ de PDIN. $3783 \mathrm{~g}$ de $P D I E, 485 \mathrm{~g}$ de MAT.

portante et significative de la composition du lait. Le taux butyreux a chuté de $4,0 \mathrm{~g} / \mathrm{kg}$ alors que le taux protéique et le taux de lactose ont augmenté respectivement de 1,0 et $0,7 \mathrm{~g} / \mathrm{kg}$ (tableau II). Le changement a été assez rapide sur le taux butyreux (maximum au cours de la deuxième semaine) mais plus lent sur te taux protéique (maximum au cours de la troisième semaine) avec un léger effet rémanent (fig 2) puisque l'effet positif du MPG sur le taux protéique semble persister après l'arrêt de MPG.

La production de matières grasses a diminué de façon importante et significative (- $88 \mathrm{~g}$ soit $11 \%$ ); par contre, celle des matières protéiques a augmenté significative- ment mais dans des proportions très modestes (+ $13 \mathrm{~g}$ soit $2 \%$ ).

La proportion des différentes fractions azotées (protéines, alactalbumine, ßlactoglobuline, caséines) n'a pas été altérée. L'accroissement du taux de caséine représente $77 \%$ de celui du taux protéique (tableau II).

Les teneurs en minéraux ont été peu modifiées à l'exception d'une légère baisse significative du $\mathrm{Ca}^{2+}$ soluble et du $\mathrm{Na}^{+}$(tableau (I).

La vitesse de coagulation, mesurée sur la courbe d'évolution de la viscosité, est augmentée de façon significative (+ $36 \%$ ) (tableau III). La viscosité maximale du lait 
Tableau II. Effet du niveau énergétique et de l'apport de monopropylène glycol sur la composition du lait.

\begin{tabular}{|c|c|c|c|c|}
\hline & Témoin négatif & Témoin positif & $M P G$ & ETR \\
\hline \multicolumn{5}{|l|}{ Productions } \\
\hline Lait $(\mathrm{kg})$ & 20,1 & 20,0 & 19,8 & 0,6 \\
\hline Matières protéiques $(\mathrm{g})$ & 617 & 616 & $629^{*}$ & 18 \\
\hline Matières grasses $(\mathrm{g})$ & 827 & 821 & $736^{* *}$ & 30 \\
\hline \multicolumn{5}{|l|}{ Compostion du lait entier } \\
\hline Taux protéique $(\mathrm{g} / \mathrm{kg})$ & 30,9 & 30,8 & 31,8 ** & 0,5 \\
\hline Taux butyreux $(\mathrm{g} / \mathrm{kg})$ & 41,4 & 41,2 & $37,3^{* *}$ & 1,2 \\
\hline Lactose $(\mathrm{g} / \mathrm{kg})$ & 52,9 & 53,1 & $53,7^{\star \star}$ & 0,5 \\
\hline \multicolumn{5}{|l|}{ Composition du lait écrémé } \\
\hline Matières azotées totales $(\mathrm{g} / \mathrm{kg})$ & 33,2 & 33,0 & $34,5 \mathrm{z}$ & 0,4 \\
\hline Protéinus $(\mathrm{g} / \mathrm{kg})$ & 31,7 & 31,5 & $33,0 \mathrm{z}$ & 0,4 \\
\hline Caséines $(\mathrm{g} / \mathrm{kg})$ & 26,4 & 26,3 & $27,4 \mathrm{z}$ & 0,4 \\
\hline Alphalactalbumine $(\mathrm{g} / \mathrm{kg})$ & 1,1 & 1,1 & 1,2 & 0,05 \\
\hline Bétalactoglobuline $(\mathrm{g} / \mathrm{kg})$ & 4,6 & 4,5 & 5,4 & 0,5 \\
\hline Calcium total $(\mathrm{g} / \mathrm{kg})$ & 1,28 & nd & 1,25 & 0,18 \\
\hline Calcium soluble $(\mathrm{g} / \mathrm{kg})$ & 0,34 & nd & 0,30 * & 0,006 \\
\hline Calcium colloïdal $(\mathrm{g} / \mathrm{kg})$ & 0,95 & nd & 0,94 & 0,19 \\
\hline Magnésium total $(\mathrm{g} / \mathrm{kg})$ & 0,11 & nd & 0,10 & 0,01 \\
\hline Sodium total $(\mathrm{g} / \mathrm{kg})$ & 0,25 & nd & $0,23 *$ & 0,001 \\
\hline Potassium total $(\mathrm{g} / \mathrm{kg})$ & 1,64 & nd & 1,61 & 0,12 \\
\hline
\end{tabular}

$*: p<0,01 ; *: p<0,05 ; 2: p<0,1 ;$ nd $:$ non disponible.

au cours de la coagulation, qui correspondrait à la fin de la phase secondaire de cette gélification, est augmentée de façon significative de 14\%; par contre, le temps nécessaire pour obtenir cette viscosité maximale n'a pas varié.

Le rendement fromager en frais a été amélioré $(+3,8 \% ; P<0,01)$. Les caractéristiques de texture ont aussi varié : la force de résistance du caillé, qui reflète sa fermeté, est plus grande.

La teneur du plasma en glucose a augmenté de $7 \%$ par rapport au témoin négatif et de $3 \%$ par rapport au témoin positif $(P<0,01)$. Le $\beta$ hydroxybutyrate n'a pas varié. Le taux d'urée a été intermédiaire entre ceux des 2 autres traitements $(P<0,01)$ (tableau IV).

\section{DISCUSSION}

\section{Niveau énergétique}

L'augmentation de l'apport des nutriments énergétiques sans changement de leur composition n'a provoqué aucune modification de la quantité de lait produite, de sa composition chimique et de ses propriétés 
Tableau III. Effets du niveau énergétique et de l'apport de monopropylène glycol sur les propriétés technologiques du lait écrémé.

\begin{tabular}{|c|c|c|c|c|}
\hline & Témoin négatif & Témoin positif & $M P G$ & ETR \\
\hline Rendement en frais (\%) & 31,1 & 29,3 & $34,0 \mathrm{z}$ & 1,3 \\
\hline Fermeté $(g)$ & 1,3 & 1,4 & $1,9^{*}$ & 0,1 \\
\hline \multicolumn{5}{|l|}{ Paramètres de coagulation } \\
\hline Viscosité maximale (mPas.s) & 3,18 & 3,12 & 3,52 * & 0,04 \\
\hline Différence de viscosité (mPa.s) & 2,01 & 1,95 & 2,34 * & 0,06 \\
\hline Temps de coagulation (s) & 986 & 1001 & 948 & 55 \\
\hline Vitesse de coagulation (mPas.s/s) & 1,35 & 1,28 & $1,70 z$ & 0,01 \\
\hline
\end{tabular}

$\because p<0,05 ; z: p<0,1$.

Tableau IV. Effet du niveau énergétique et de l'apport de MPG sur les paramètres sanguins.

\begin{tabular}{lcccc}
\hline & Témoin négatif & Témoin positif & MPG & ETR \\
\hline Glucose $(\mathrm{mg} / 100 \mathrm{ml})$ & 65,0 & $67,6^{* *}$ & $69,5^{* *}$ & 2,5 \\
Urée $(\mathrm{mg} / 100 \mathrm{ml})$ & 30,4 & $25,0^{* *}$ & $28,2^{* *}$ & 2,4 \\
Bhydroxybutyrate $(\mathrm{mg} / 100 \mathrm{ml})$ & 6,0 & 5,9 & 5,9 & 1,2 \\
\hline
\end{tabular}

$* *: p<0,01$.

technologiques. Généralement, cela se traduit par un accroissement de la teneur en protéines (Remond, 1985) et de la production de lait, qui auraient pour origine le supplément de nutriments disponibles et le changement de leur nature (Armstrong et Prescott, 1971).

Dans notre essai, l'absence d'effets peut être liée au fait que :

- le régime témoin négatif couvrait déjà tous les besoins et que le supplément effectivement apporté était faible (+ 1,3 UFL) et :
- que la fourniture en acides aminés (PDI) est restée strictement constante (l'augmentation des protéines microbiennes étant compensée par une réduction du supplément protéique) contrairement à la plupart des essais antérieurs.

Les variations de la teneur en urée dans le sang ont pour origine le déséquilibre énergie-azote dans la ration; le témoin négatif a reçu une trop grande quantité d'azote par rapport à l'énergie fournie. Cela a entraîné une augmentation de l'urée dans le sang. 
Tableau V. Effet du monopropylène glycol sur les caractéristiques digestives ruminales (moyennes des valeurs entre 0 et $9 \mathrm{~h}$ après le repas).

\begin{tabular}{lrr}
\hline & $\begin{array}{c}\text { Témoin } \\
\text { négatif }\end{array}$ & MPG \\
\hline $\mathrm{pH}$ & 6,75 & 6,77 \\
Concentration en AGV (mmol/) & 77,6 & 71,8 \\
Composition en AGV (mol 100) & & \\
Acide acétique & 67,0 & 56,7 \\
Acide propionique & 19,5 & 25,5 \\
Acide isobutyrique & 0,8 & 2,8 \\
Acide butyrique & 9,6 & 8,8 \\
Acide isovalérique & 1,3 & 2,7 \\
Acide valérique & 1,2 & 2,3 \\
Acide caproique & 0,6 & 1,2 \\
\hline
\end{tabular}

a

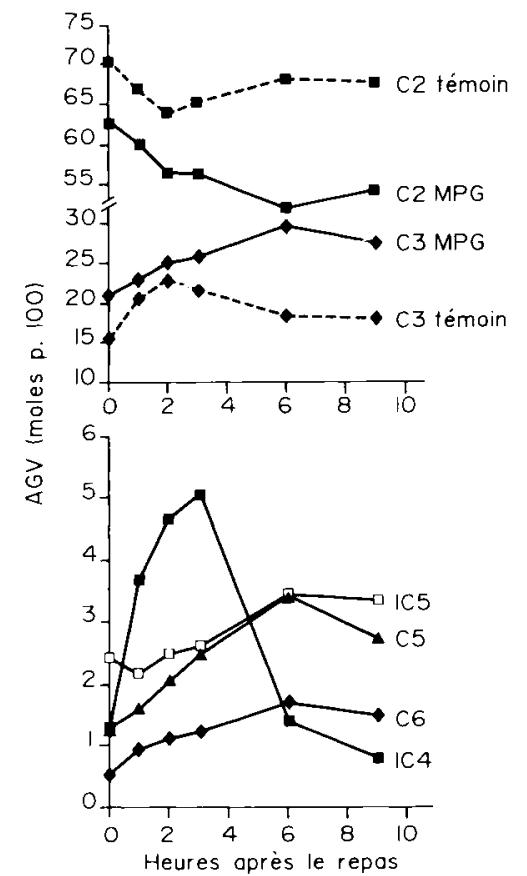

Fig 1. Évolution de la proportion relative des acides gras volatils dans le jus de rumen au cours de la journée. a) Comparaison de l'évolution post-prandiale de la teneur en acide acétique et en acide propionique entre le témoin et le traitement MPG. b) Évolution de la proportion relative des acides gras volatils minéraux au cours de la journée après injestion de monopropylène glycol.

\section{Monopropylène glycol}

L'effet glycogénique du MPG, observé depuis longtemps chez les ruminants (Emery et al, 1964; Czerkawski et Breckenbridge, 1972; Clapperton et Czerkawski, 1972) a été retrouvé ici à travers l'augmentation importante du propionate ruminal et du glucose sanguin. D'après l'évolution postprandiale du $\mathrm{pH}$ et de l'acidité volatile, la dégradation ruminale serait lente et seulement partielle puisque le MPG apporté en supplément n'a pas augmenté la teneur en acides gras volatils. Ceci serait en accord avec les résultats de Gieseke (1974) pour qui les 2 voies d'utilisation du MPG (absorption directe et dégradation dans le rumen) seraient à peu près de même importance. Cependant, cette répartition est très variable (Rémond et al, 1984) et la forte dose employée ici pourrait être une source de variation supplémentaire. Par ailleurs, les accroissements très importants des AGV en $\mathrm{C} 5$ et $\mathrm{C} 6$ n'ont pas encore été signalés et il serait important d'en connaître les raisons et surtout l'effet sur le métabolisme.

L'apport de monopropylène glycol dans l'alimentation n'a eu aucun effet sur la quantité de lait produite. Ceci est en opposition avec beaucoup de résultats antérieurs (Emery et al, 1964; Hooven et al, 1969; Fisher et al, 1971 et 1973; Sergheraert et Rémond, 1983 cités par Rémond et al, 1984). Un effet positif est attendu surtout en cas de cétoses cliniques ou subcliniques (Hooven et al, 1969; Fisher et al, 1973). Or, ce n'était pas le cas dans notre essai : animaux primipares après le pic de lactation, avec un bilan énergétique équilibré et pour lesquels la teneur sanguine en Bhydroxybutyrate était faible et celle en glucose normale. Au contraire, la dose très élevée de MPG aurait pu provoquer comme chez Fisher et al (1973) une légère baisse de la production; la produc- 


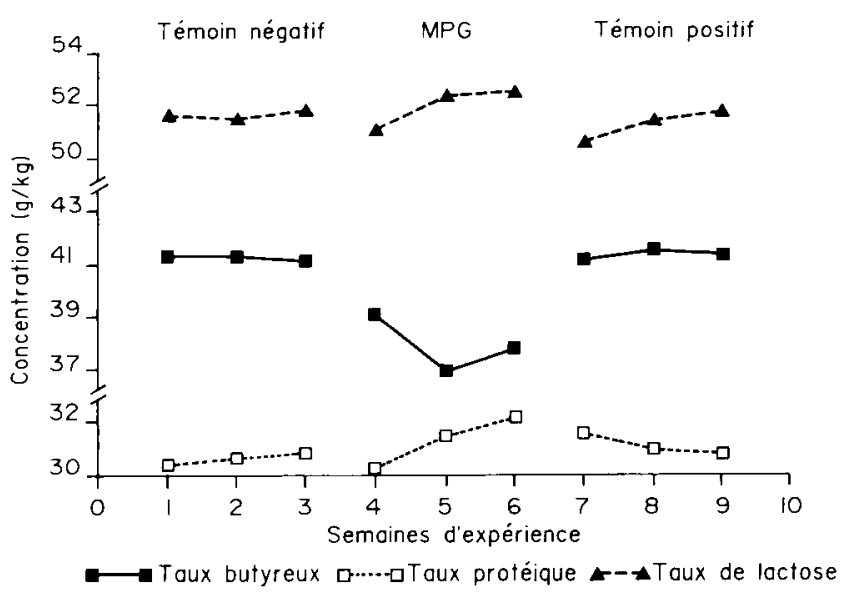

Fig 2. Evolution des teneurs du lait en matières grasses, en protéines et en lactose pendant les 3 traitements (Chaque lot a reçu les 3 traitements mais à des périodes différentes. Ces points ont donc été obtenus en faisant la moyenne des 3 résultats concernant un traitement réalisé à des périodes différentes pour chacun des lots).

tion de lait journalière observée a tendance à diminuer lors des derniers jours de traitement mais ce n'est pas net, car la durée de traitement était trop courte pour bien observer ce phénomène. L'absence de réponse de la production laitière à l'apport de MPG, précurseur d'acide propionique, serait due à une utilisation préférentielle de l'énergie pour la synthèse de tissus corporels aux dépens de celle du lait (Ørskov et al, 1969; Holter et al, 1972) d'autant plus que l'afflux supplémentaire de glucose aura accru le taux d'insuline (Waldo et Schultz, 1960).

La baisse importante du taux butyreux et l'augmentation du taux protéique que nous avons observées sont en accord avec les essais antérieurs (Rémond et al, 1984) qui montrent les variations antagonistes de ces 2 paramètres (fig 1). Les réponses plus importantes dans notre essai sont probablement dues à la dose plus élevée de MPG (2-8 fois plus).
Une telle baisse du taux butyreux est classiquement observée avec d'autres méthodes conduisant à un accroissement de la fourniture en propionate et en glucose (apport de concentrés riches en amidon ou infusions digestives) mais un fait remarquable ici est la bonne homogénéité des réponses du taux butyreux d'un animal à l'autre. Les mécanismes en cause pourraient être ceux classiquement avancés dans ces situations (Williams et Elliot, 1980) : d'une part, la réduction de la fourniture ruminale d'acétate a pu limiter la synthèse mammaire de novo d'acides gras courts et d'autre part, l'augmentation observée de fourniture de propionate et de glucose aurait accru, via la sécrétion plus élevée d'insuline, l'activité métabolique de dépôt de tissu adipeux. Ceci pourrait conduire à une diminution de la disponibilité mammaire en acides gras non estérifiés, sources des acides gras longs du lait, comme il a souvent été noté avec le MPG 
(Sauer et al, 1973). Cependant, l'action insulinosécrétrice pourrait être plus modérée que celle d'autres sources glucidiques (Hamada et al, 1982), d'autant plus que l'action du MPG est lente est durable au cours de la journée.

Néanmoins, globalement, l'approvisionnement énergétique de la mamelle n'aurait pas été affecté par la disponibilité supplémentaire de glucose. L'effet positif sur le taux de lactose (compensé par la chute du sodium) en serait la conséquence.

L'accroissement du taux protéique $(1 \mathrm{~g} /$ kg) observé avec le MPG par rapport à celui du témoin isoénergétique, ne semble pas lié ici à la réduction de la sécrétion d'eau. II est analogue à l'effet obtenu avec des infusions de propionate (Elliot, 1980; Emmanuel et Kennelly, 1984) et de glucose (Vik-Mo et al, 1974). Le mécanisme d'action n'est pas connu : le glucose agit-il directement grâce à l'augmentation de sa disponibilité mammaire ou indirectement, via le profil hormonal, ou bien la disponibilité mammaire en acides aminés a-t-elle été modifée (synthèse protéique ruminale ou épargne métabolique de certains acides aminés) ?

L'apport de monopropylène glycol n'a pas modifié les différentes fractions azotées du lait. Cette absence de modification confirme les résultats récapitulés par Rémond (1985) selon lesquels la proportion des caséines dans les protéines ne varie pas avec le niveau et la nature des apports énergétiques. Cependant, les aptitudes technologiques du lait sont améliorées lorsque les vaches consomment du monopropylène glycol. La vitesse d'agrégation des micelles est plus rapide, ce qui entraîne une fermeté du gel plus grande. Cela va donc dans le sens de l'amélioration des aptitudes fromagères du lait : le travail du caillé est facilité et l'égouttage se fait plus rapidement.
En conclusion, l'augmentation de l'apport des nutriments énergétiques sans changement de leur composition n'a pas modifié la production et la composition du lait, alors que le monopropylène glycol, fournisseur en composés glycogéniques, donc modifiant la fourniture des nutriments énergétiques par rapport au etémoin positif» a entraîné des modifications non négligeables de la composition du lait : le rapport (taux protéique / taux butyreux) est fortement augmenté $(0,75-0,85)$ par suite des effets antagonistes sur les 2 composants. Son mécanisme d'action mérite d'être approfondi car il semblerait, au vu d'essais réalisés ultérieurement, que l'augmentation de la teneur en acide propionique du rumen ne soit pas à l'origine de toutes les variations de composition. Une étude plus fine des paramètres fermentaires du rumen liée à une analyse des paramètres sanguins et des flux digestifs, permettrait d'avoir une meilleure idée du mode d'action de ce produit.

\section{REFERENCES}

Armstrong DG, Prescott JH (1971) Amount, physical form and composition feed and milk secretion in the dairy cow. In: Lactation (I Falconer, ed) Butterworths, Londres, 349-378

Auriol P, Jarrige R (1962) Possibilités de modifier la composition du lait. Int Dairy Annu Bull 2, 1-39

Clapperton JL, Czerkawski JW (1972) Metabolism of propane-1:2-diol infused into the rumen of sheep. Br J Nutr 27, 553-560

Czerkawski JW, Breckenbridge G (1972) Fermentation of various glycolytic intermediates and other compounds by rumen microorganisms, with particular reference to methane production. Br J Nutr 27, 131-146

Elliot JM (1980) Digestive physiology and metabolism in ruminant. Westport, AVI Publishing Company, 485-503

Emery RS, Burg N, Brown LD, Blank GN (1964) Detection, occurence and prophylactic treat- 
ment of borderline ketosis with propylene glycol feeding. J Dairy Sci 47, 1074-1079

Emmanuel B, Kennelly JJ (1984) Effect of propionic acid on ketogenesis in lactating sheep fed restricted rations or deprived of food. J Dairy Sci 67, 344-350

Fisher LJ, Erfle JD, Sauer FD (1971) Preliminary evaluation of the addition of glucogenic materials to the rations of lactating cows. Can $J$ Anim Sci 51, 721-727

Fisher LJ, Erfle JD, Lodge GA, Sauer FD (1973) Effects of propylene glycol or glycerol supplementation of the diet of dairy cow on feed intake, milk yield and composition and incidence of ketosis. Can J Anim Sci 53, 289 296

Giesecke D (1974) Metabolic kinetics of the anti-ketotic compound 1,2-propanediol in the rumen. Arch Int Physiol Biochem 82, 645-649

Hamada T, Ishii T, Taguchi S (1982) Blood changes of spontaneously ketotic cows before and four hours after administration of glucose, xylitol, 1,2-propanediol, or magnesium propionate. J Dairy Sci 65, 1509-1513

Holter JB, Jones LA, Colovos NF, Urban WE (1972) Caloric value of acetate and propionate for lactating dairy cows. J Dairy Sci 55 , 1757-1762

Hooven NW, Plowman RD, Smith JW (1969) Efficacy of feeding propylene glycol to reduce the incidence of severity of ketosis. $J$ Dairy Sci 52, 915

Korolczuck J, Maubois JL (1987) Computerized viscosimetric method for studying rennet coagulation of milk. $J$ Text Stud 18, 157-172

Orskov ER, Flatt WP, Moe PW, Munson AW, Hemken RW, Katz J (1969) The incidence of ruminal infusion of volatile fatty acids on milk yield and composition and on energy utilization by lactating cows. Br J Nutr 23, 397-405

Rémond $B$ (1985) Influence de l'alimentation sur la composition du lait de vache -2 - Taux protéique : facteurs généraux. Bull Tech CRZV 62, 53-67

Rémond $B$, Remesy $C$, Ruffio $P$, Chilliard $Y$ (1984) Intérêt du monopropylène glycol dans la prévention et dans le traitement des cé- toses chez les vaches laitières. Bull Tech CRZV 56, 21-30

Rook JAF, Bajch CC (1961) The effects of intraruminal infusions of acetic, propionic and butyric acids on the yield and composition of the milk of the cow. Br J Nutr 15, 361-369

Rook JAF, Balch CC, Johnson VM (1965) Further observations on the effects of intraruminal infusions of volatile fatty acids and of lactic acid on the yield and composition of the milk of the cow. Br J Nutr 19, 93-99

Rulquin H (1982) Effets sur la digestion et le métabolisme des vaches laitières d'infusions d'acides gras volatils dans le rumen et de caséinate dans le duodénum - I - Production et digestion. Reprod Nutr Dev 22, 905-921

Sauer FD, Erfle JD, Fisher LJ (1973) Propylene glycol and glycerol as a feed additive for lactating dairy cows: an evaluation of blood metabolite parameters. Can J Anim Sci 53, 265271

Talke H, Schubert GE (1965) Enzymatische Harnstoffbestimmung in Blut und Serum in optischen Test nach Warburg. Klin Wochenschr 43, 174

Thomasow J, Voss E (1977) Méthode pour la détermination de la fermeté du coagulum du lait. Bull Annu FIL-IDF 99, 1-12

Trinder $P$ (1969) Determination of blood glucose using an oxydase peroxydase system with a non carcinogenic chromogen. Ann Clin Biochem 6, 24-27

Vik-Mo L, Emery RS, Huber JT (1974) Milk protein production in cows infused abomasally with casein or glucose. J Dairy Sci 57,869 877

Waldo DR, Schultz LH (1960) Blood and rumen changes following the intra-ruminal administration of glycogenic materials. J Dairy Sci 43, 496-505

Williams JA, Elliot M (1980) Mammary extraction of propionate in lactating cows. $J$ Dairy $\mathrm{Sci}$ $63,1999-2005$

Williamson $\mathrm{DH}$, Mellanby $\mathrm{J}$ (1974) D-(-) 3hydroxybutyrate. in Bergmeyer $\mathrm{HU}, \mathrm{Me}$ thods of enzymatic analysis, vol 4, Academic Press, New-York, 1836-1839 